\title{
Gold Recovery From Used Electrical Contactors
}

\author{
John S. Niederkorn and Steve Huszar \\ Ultra Instrument Laboratory, Inc., Dallas, Texas, U.S.A. \\ During the last 15 years about 700 t of gold have been consumed in the manufacture of low \\ voltage electrical contactors, which, over a period of time, have become redundant and thus \\ the gold component bas become candidate for recovery. The results of laboratory and batch \\ pilot experimentation, reported in this article, bave demonstrated an average gold recovery \\ rate of 95 per cent from used contactors by means of mechanical and chemical processing.
}

Due to its excellent electrical conductivity and resistance to corrosion, gold is widely used in the electronics industry in particular to ensure high reliability, where required, of the different types of connectors, printed circuits, relays, switches and other devices. The application of gold for low voltage contact surfaces where insulating oxide tarnish film, or friction polymer formation should be excluded - is the only technical solution which has served the test of time and is not shown to be wanting.

During the last three decades considerable quantities of gold have been used in the electronics industry. According to the

Fig. 1. A widely used type of low voltage telephone contactor
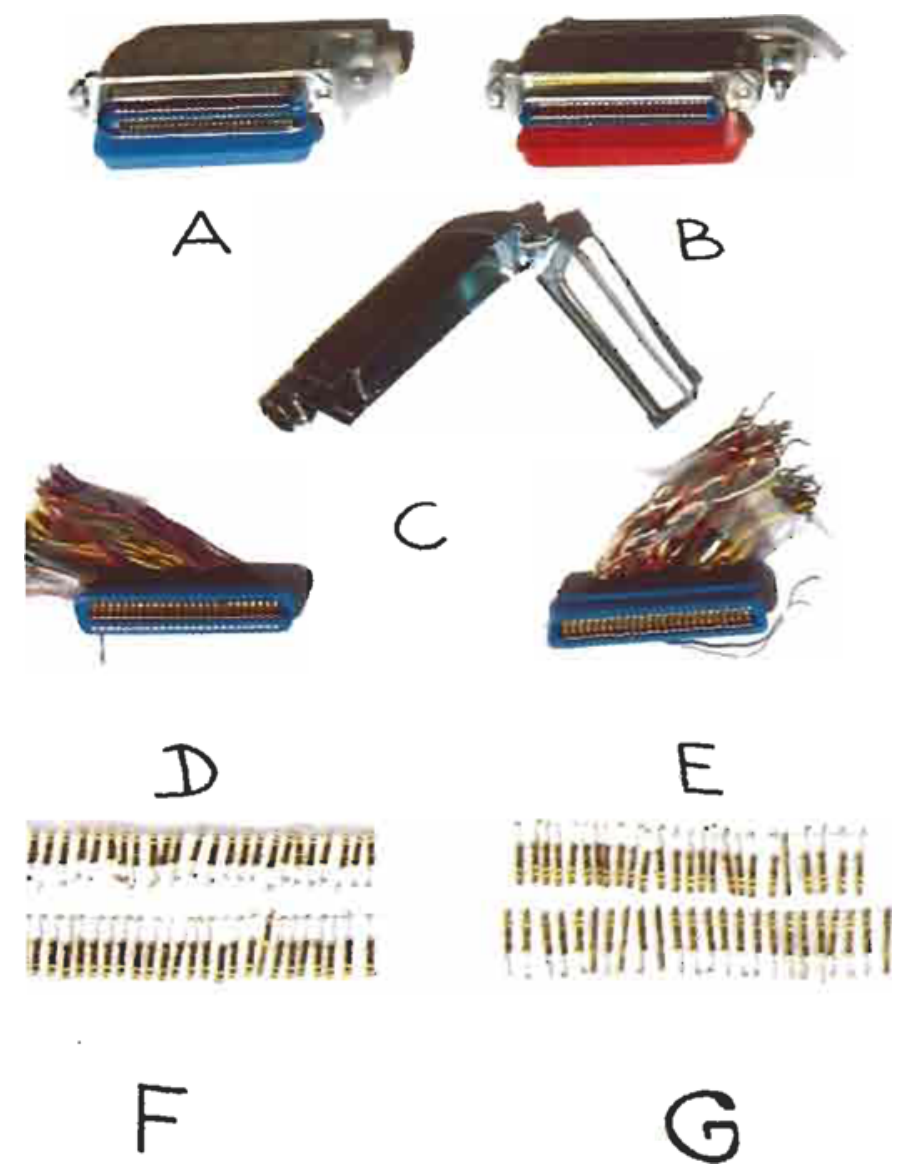

published data $(1,2,3)$ in 1968,82 t of gold were consumed by the industry in the U.S.A. and Western Europe. The peak consumption year was 1973 when $127 \mathrm{t}$ had been used after which decline in consumption reduced gold usage to $67 \mathrm{t}$ in 1975. Since 1978, the annual usage of gold for electronics applications has been fairly stable at about 80 t per year for the two markets mentioned.

It is significant to point out that both in the U.S.A. and Western Europe about 40 per cent of the total electronic consumption of gold covers the different type of connectors, which constitute the most widely spread gold-containing type of electronic device.

\section{Used Electronic Devices as a Source of Gold Recycling}

It is very difficult to say how much of the approximately 1400 t of gold used in the electronics industries in the Western world over the past 2 decades would be available for recycling and how much has already been recovered from the used electronic parts. There is also little available published information on the gold recovery technologies used for electronic waste materials especially on an industrial scale. It is clear, however, that a number of technological alternatives exist which might be applied in an attempt to produce an economically justified gold recovery process from this type of redundant material (4-7).

\section{A Possible Gold Recovery Process}

Figure 1 shows the various parts of a type of low voltage telephone contactor pair, $A$ and $B$, in widespread use. Each of them contains 50 gold plated contact units, $F$ and $G$, which are fixed in plastic contact holders, D and $\mathrm{E}$. An insulated wire is soldered to each contact unit. The plastic contact holders are encapsulated in a zinc coated steel case, $\mathrm{C}$.

The purpose of the investigation reported herein was to find a technologically and economically acceptable way to recover gold from the contactors shown in Figure 1, positions $A$ and $B$.

As a first step, the average quantity of gold per contactor was determined. For this purpose 10 contactors were chosen randomly from a batch of one thousand. Each of them was opened and the individual contact units ( $F$ and $G$ in Figure 1) were removed, separated from the wire ends and analyzed for gold content.

Table I shows the results of the chemical analysis. The gold quantity indicates the total amount of gold found in fifty contact units'which were fixed in each of the individual contactors. The average of $40.1 \mathrm{mg}$ gold per contactor can be considered 
representative, as a result of the ten analyzed samples being randomly selected from the total of one thousand contactors and in view of the low spread in the gold contents obtained.

The weight of 50 contact elements was found to be $3850 \mathrm{mg}$ leading to an average gold content of 1.04 per cent.

In spite of the fact that contact elements with copper substrates are relatively rich in gold, their removal from the plastic supports for gold recovery would be a very expensive procedure. Therefore, gold recovery should be ideally achieved without their removal.

\section{Gold Recovery by Using Nitric Acid}

From the results of the above preliminary tests it became obvious that without the separation of plastic holders from the metal case, gold cannot be effectively recovered from this type of contactor.

Due to the porosity level of such gold deposits, the copper base metal can be dissolved with relative ease in dilute nitric acid. With 45 to 50 per cent nitric acid solution the base metal was completely dissolved in $40 \mathrm{~min}$. The undissolved residue contained 99.6 per cent gold as a very fine film.

\section{Pilot Test Operation}

Three hundred plastic contact-holders were introduced in a plastic vessel ( $A$ in Figure 2 ), covered with a lid, which contained an inlet for acid introduction and an outlet for nitrogen dioxide exit. At the bottom of the vessel there was a third outlet for the used acid and the release of the residual gold film. A riddled plate $B$ at the bottom $C$ served for the separation of the gold film from the mass

\begin{tabular}{c|c|c}
\multicolumn{2}{c}{$\begin{array}{c}\text { Table I } \\
\text { The Surface Tensions of Liquid Gold }\end{array}$} \\
\hline $\begin{array}{c}\text { Temperature, } \\
{ }^{\circ} \mathrm{C}\end{array}$ & Atmosphere & $\begin{array}{c}\gamma_{\mathrm{Au}}^{\mathrm{L}} \\
\mathrm{mJ} / \mathrm{m}^{2}\end{array}$ \\
\hline 1108 & & $1130 \pm 10$ \\
1200 & Argon & 1070 \\
1300 & Helium & 1020
\end{tabular}

\section{Gold Recovery Direct from Encapsulated Contactors}

Encapsulated contactors within their zinc-coated steel cases were treated with sodium cyanide at different temperatures. It was established that the gold dissolution rate was very low and complete dissolution of gold could be achieved only with a huge consumption of cyanide. The explanation of this phenomenon is related to the presence of large quantities of zinc and steel (iron) in the reaction medium.

Similar experiments were carried out using solutions of thiourea at concentrations of 10 to $50 \mathrm{~g} / \mathrm{l}$ acidified by sulphuric acid to $\mathrm{pH} 1$ and containing ferric sulphate as oxidant in place of sodium cyanide for gold dissolution. In this case it was also established that the gold dissolution rate was very low and complete dissolution of gold could not be achieved because the $\mathrm{pH}$ of the solution could not be controlled. This can also be explained by the presence of large quantities of zinc and iron in the reaction medium.
Fig. 2. Schematic diagram of recovery process developed in the work reported in this article.

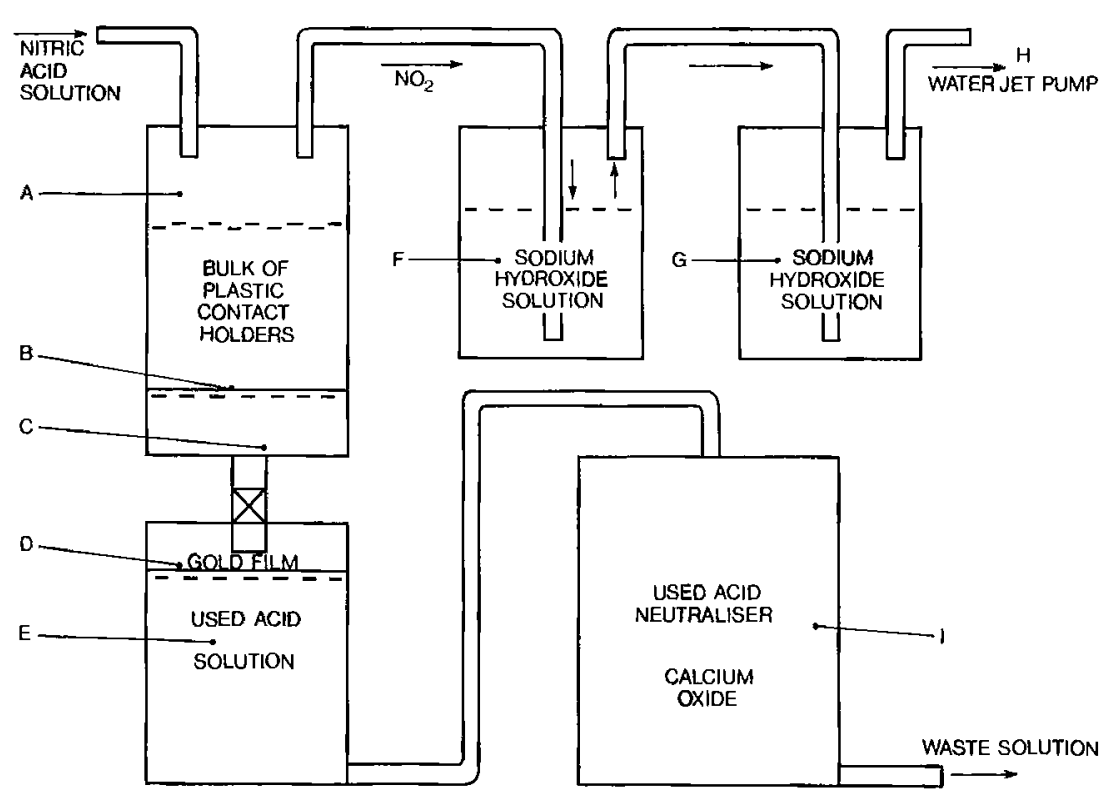


of plastic contact holders.

The entire contact holder bulk was covered with 45 percent nitric acid, upon which base metal dissolution began, this being accomplished within 40 to $50 \mathrm{~min}$. The released nitrogen dioxide gas was sucked by a water jet suction pump $\mathrm{H}$ through two absorption vessels $F$ and $G$, containing concentrated sodium hydroxide solution. Following the base metal dissolution, the used acid with the gold film suspension was released through a plastic sieve $D$. The gold film remained on the sieve and the used acid was collected on the bottom of the vessel $E$. The used acid could be neutralized with quick lime in vessel $\mathrm{I}$.

This procedure guarantees a 100 per cent chemical recovery of gold. However, the overall gold recovery does not exceed 95 per cent due to the mechanical losses of gold film through the separation sieve and in the lining of the plastic contact holders. The final gold recovered from 300 processed contactors amounted to 11.0 to 11.3 $\mathrm{g}$ of pure gold in film form.

\section{Concluding Remarks}

By applying the procedure discussed above, the economic opportunity of gold recovery from low voltage contactors as described is very encouraging, but depends very much on the cost of the operations involved in the opening of the metal cases and removal of wire ends.

The acid consumption and chemical operation costs represent 35 to 40 per cent of the total operating costs.

It goes without saying that on a large industrial scale the economic effects would be much more beneficial than on batch pilot plant scale. However, even in the U.S.A. at the present gold price levels the regional availability of used contactors would not appear to justify the setting up of an industrial recovery plant.

A well-organized and coordinated collection programme for gold-containing electronic waste material carried out by companies may well be a promising alternative for the purpose of gold recycling.

\section{References}

1 T.D. Cooke, Gold Bull., 1982, 15, (2), 38-42

2 Ch. Glynn and R. Conley, Gold Bull, 1979, 12, (4), 134-139

3 M. Antler, Gold Bull., 1971, 4, (3), 42-46

4 F. Ambrose and B.W. Dunning Jr., 'Precious Metals Recovery from Electronic Scrap' in 'Proc. Miner. Waste Util. Symp.', 1980, pp. 184-197

5 D. Takacs et al., 'Industrial Gold Recovery, Especially from Scrap-Electric-Circuit Boards', Hung. Pat. C1 C22B11/040, June 28, (1980)

6 F. Ambrose and B.W. Dunning, Jr., 'Mechanical Processing of Electronic Scrap to Recover Precious Metal-Bearing Concentrates' in 'Precious Metals', edited by R.O. McGachie and A.G. Bradley, Pergamon Press Canada Ltd., 1981, pp. 67-76

7 J.R. Valentine, et al., 'A pparatus and Method for Recovery of a Precious Metal in Pure or Alloyed Form from a Bimetallic Material', Ger. Offen. 3037 112(1981)

\section{Gold Cluster Labelling for Electron Microscopy}

\section{HIGH RESOLUTION MARKING OF CARBOHYDRATE SITES ON GLYCOPROTEINS}

Scanning transmission electron microscopes (STEM) are able to image single heavy metal atoms due first to the high contrast and high electron collection efficiency of the dark field annular collector of the system, and secondly to the high resolution which can be obtained with a field emission gun coupled to low-operation lenses. As a result of this facility biologists have shown much interest in using STEM to 'spot' heavy atom markers attached to organic molecules to help in structural studies. Such labelling can be carried out with a high degree of selectivity or specificity.

The application of single heavy metal atoms to label isolated noncrystalline macromolecules has been limited by a number of significant obstacles. In particular the heavy electron dose required for reliable heavy atom detection $\left(10^{3}\right.$ to $\left.10^{5} \mathrm{e} / \AA^{2}\right)$ is considerably higher than that at which most biological molecules suffer damage ( 1 to $10 \mathrm{e} / \AA$ ). Furthermore, single heavy atoms have been observed to migrate following radiation damage which would tend to reduce the level of specificity achieved.

The use of gold colloids has gone some way towards overcoming the above limitations. Another promising avenue has been the use of stable complexes or clusters of heavy atoms and, to this end, the reported application of an undecagold phosphine cluster $[(p-$ $\left.\left.\mathrm{H}_{2} \mathrm{NCH}_{2} \mathrm{C}_{6} \mathrm{H}_{4}\right)_{3} \mathrm{P}_{7} \mathrm{Au}_{11}\right]^{3+}$ containing a central core of 11 gold atoms surrounded by an organic 'shell' of total radius of about $10 \AA$ J.S. Wall et al., Ultramicrosc., 1982, 8, 397-402) is particularly exciting.

A number of important advantages result from marking with the undecagold phosphine cluster instead of with a single heavy,metal atom. In particular the gold core can be located in the electron microscope at a much lower electron dose $\left(50 \mathrm{e} / \AA^{2}\right)$, and the cluster shows very little movement upon irradiation - gradual decomposition does however take place with loss of half its intensity at a dose of some $10^{3} \mathrm{e} / \AA^{2}$. The resolution which the gold cluster allows is 5 to 10 times better than that achieved with conventional markers such as ferritin (diameter $120 \AA$ ) or ferritin-antibody. Furthermore the surrounding organic 'shell' contains 21 amino groups which can be cross-linked directly or chemically modified, and by suitable organic reactions covalent bonding to specific functional groups on the substrate molecule to be labelled is possible.

More recently (JJ. Lipka et al., J. Ultrastruct. Res, 1983, 84, 120-129) the specific labelling was reported of carbohydrate moieties on the glycoprotein human haptoglobin $(\mathrm{Hp})$ in the haptoglobinhaemoglobin complex (Hp.Hb) which is readily observed in the electron microscope as a barbell shaped molecule.

The carbohydrate moieties of the Hp.Hb complex were oxidized by sodium periodate to produce aldehydes. The primary amines on the undecagold cluster were allowed to react with aldehyde residues to produce Schiff's base linkages which were subsequently reduced with sodium borohydride. High-resolution STEM showed the undecagold label to be localized in a region known to be occupied by the heavy chains of haptoglobin with which the carbohydrate is exclusively associated. The amount of labelling was found to be two to four gold clusters per molecule when excess label was reacted. This report concludes that the high chemical specificity of the reaction and the high resolution of the gold clusters should make this new label of widespread value in studies of other glycoproteins or carbohydrate-bearing molecules.
C.I. 